\title{
Effects of altered preload on left ventricular systolic time intervals in acute myocardial infarction'
}

\author{
Purshottam K. Khanna, Pravin M. Shah, ${ }^{2}$ David H. Kramer, Richard A. Schaefer, and Ira Tager \\ From the Cardiology Unit, Department of Medicine, University of Rochester School of Medicine and Dentistry, \\ Rochester, New York, U.S.A.
}

\begin{abstract}
Acute effects of altered preload on systolic time intervals were examined in 17 patients; 7 subjects without acute myocardial infarction and normal haemodynamics (post aortic valve surgery-Class I), 6 patients with acute myocardial infarction and raised left ventricular filling pressure, and 4 patients with acute myocardial infarction and normal filling pressure. Alterations in preload were brought about by applications of venous cuffs on both thighs. Observations were also made on the systemic artery and pulmonary artery pressures, cardiac output, and heart rate before and after application of venous occlusive cuffs. In Class I postsurgical patients with normal haemodynamics, pre-ejection period (PEP) lengthened, left ventricular ejection time (LVET) shortened, and PEP/LVET ratio was increased after application of thigh cuffs. While heart rate was held constant by atrial pacing, stroke index decreased, pulmonary artery systolic and diastolic pressures fell, but there was no significant change in systemic artery pressure. In patients having acute myocardial infarction with raised left ventricular filling pressure, $P E P$ was shortened, $L V E T$ remained unchanged, and $P E P / L V E T$ ratio decreased after application of venous cuffs. No significant changes in heart rate, cardiac output, and systemic artery pressures were observed. However, both the pulmonary artery systolic and diastolic pressures fell. In patients having acute myocardial infarction with normal left ventricular filling pressure, there was no significant change in any of the aforementioned parameters. These differences in responses to applications of venous occlusive cuffs are explained on the basis of alterations in left ventricular performance. It is concluded that in patients with acute myocardial infarction, observations on the effects of altered preload on systolic time intervals may provide a more accurate index of left ventricular performance than measurements of systolic time intervals alone.
\end{abstract}

Weissler, Peeler, and Roehll (196I) have proposed the use of left ventricular systolic time intervals as indirect indices of myocardial performance. There has been some divergence in the results of several studies of their value as indicators of ventricular function in acute myocardial infarction (Diamant and Killip, 1970; Habte-Michael, Gould, and Gomprecht, 197I). Pre-ejection period (PEP) may be either normal or prolonged, while LV ejection time (LVET) is generally shortened (Samson, 1970; Bennett, Smithen, and Sowton, 1970). PEP/LVET ratio has been reported to correlate with ejection fraction in patients with

Received 2 May 1973.

1 This work was carried out during the tenure of American Heart Association Teaching Scholar Award in Cardiology.

2 Supported by MIRU contract and Grants-in-Aid from the National Heart and Lung Institute, National Institutes of Health, Bethesda, Maryland, U.S.A. various types of cardiovascular disease (Garrard, Weissler, and Dodge, 1970). These parameters, however, do not separate patients with acute myocardial infarction from those admitted with obscure chest pain. Considerable overlap is also noted with normal subjects, so that the diagnostic use of these measurements is of relatively little value (Hodges et al., 1972). Their value in prognosis is also not uniformly accepted (Diamant and Killip, 1970; Hodges et al., 1972). Diamant and Killip (1970) reported significant correlation of abnormal time intervals with the severity of illness in acute myocardial infarction and with in-hospital mortality; while Hodges et al. (1972) were unable to confirm this in their patients. This is not altogether surprising, since in addition to the initial insult, numerous influences such as catecholamine and other hormonal production, sympathetic tone, and drugs tend to alter myocardial performance in 
different ways (Seifen, Flacke, and Alper, 1964; Shiner, Harris, and Weissler, I969; Weissler et al., 1965). Shah et al. (1969) found that systolic time intervals in subjects with normal haemodynamics behave in a predictable manner following alterations in preload when the heart rate was held constant. It was suggested that the response of systolic time intervals to altered preload may provide a more reliable index of myocardial function than resting values alone, since the response of functionally normal left ventricle to alterations in end-diastolic volume is different from the response of functionally compromised left ventricle. This concept was supported by the studies of Stafford, Harris, and Weissler (1970) who obtained systolic time intervals before and after vertical tilt. They reported that 3 patients in congestive heart failure responded differently from 15 normal subjects.

The present study was undertaken to assess the changes in the systolic time intervals following changes in preload induced by applications of venous cuffs, $(I)$ in patients with acute myocardial infarction with and without left ventricular dysfunction, and (2) to compare the response in patients with acute myocardial infarction with that observed in patients with normal haemodynamics (Class I-postsurgical patients).

\section{Subjects and methods}

Three groups of patients were studied. The first group (Group A) consisted of 7 male patients, aged 43 to 49 years, studied in the cardiac catheterization laboratory. These patients were studied I month to 2 years after replacement of the aortic valve with a Starr-Edwards prosthetic valve. None of the 7 had clinical evidence of valvular regurgitation or of heart failure. All were in functional class I and had a pulmonary artery diastolic pressure less than $15 \mathrm{mmHg}$. The second and third groups consisted of Io patients with acute myocardial infarction, ages 50 to 70 years, studied in the myocardial infarction research unit. Two were in pulmonary oedema and 4 had $S_{3}$ gallops and pulmonary râles. These 6 patients (Group B) had a pulmonary artery diastolic pressure more than $15 \mathrm{mmHg}$. Four patients (Group C) were uncomplicated, and each had a pulmonary artery diastolic pressure $15 \mathrm{mmHg}$ or less.

All three groups of patients had right heart catheterization with a catheter placed in the pulmonary artery. In patients of group A a No. 7 Cournand catheter was used, while in the patients of groups B and C a No. 5 Swan-Ganz flow-directed catheter was employed. A No. 18 Longdwell polyethylene cannula was introduced in the brachial artery. An atrial pacing catheter was placed in the coronary sinus.

A Sanborn contact microphone was placed over the third left interspace to record heart sounds and a Cardiocontrol low frequency microphone was used for the carotid arterial pulse trace. The outputs were fed either into a Brush (Model II-4307-00) heart sound preamplifier or a Hewlett-Packard (Model 350-1700) $\mathrm{D} / \mathrm{C}$ preamplifier. The phonocardiogram, carotid arterial pulse wave, and electrocardiogram were recorded simultaneously with a paper speed of $100 \mathrm{~mm} / \mathrm{sec}$. Systemic arterial and pulmonary artery pressures were recorded on the direct writing Brush recorder through Statham pressure transducers $\left(\mathbf{P}_{23} \mathrm{~dB}\right)$. Pulmonary wedge pressure was measured initially and was found to be almost identical to pulmonary artery diastolic pressure in each patient. The electrocardiogram, phonocardiogram, and carotid arterial pulse waves were simultaneously recorded with each cardiac output determination.

Cardiac output was determined in duplicate by indicator dilution technique with indocyanine green as the indicator. Systolic time intervals were measured at the following points in the protocol.

\section{Group A (non-myocardial infarction-func-} tional class I)

I) After placement of catheters.

2) Five minutes after atrial pacing at a rate varying between 84 and 109 beats a minute.

3) Ten minutes after inflation of blood pressure cuffs on both thighs with pressure raised to the level of systemic arterial diastolic pressure but with heart rate held constant.

4) The cuffs were then deflated and measurements repeated.

\section{Groups $B$ and $C$ (acute myocardial infarction)}

I) After placement of catheters, generally on the first day in hospital. All the patients were admitted within I8 hours of presumed occurrence of acute infarction.

2) Ten minutes after inflation of blood pressure cuffs on both thighs with pressure raised to the level of systemic arterial diastolic pressure.

3) The cuffs were then deflated and measurements repeated.

After each cardiac determination, the following systolic time intervals from ro consecutive cardiac cycles were computed and averaged. The $\mathrm{QS}_{2}$ interval, which estimates the total electromechanical systole, was measured from the onset of the $Q$ wave to the aortic component of the second heart sound. The left ventricular ejection time (LVET) was measured from the onset of the rapid upstroke of the carotid arterial pulse to its dicrotic incisura. The pre-ejection period (PEP) was calculated by subtracting the LVET from the $\mathrm{QS}_{2}$ interval. Measurements were made to the nearest $5 \mathrm{msec}$. Since the heart rate was held constant in group $A$ and the acute intervention did not result in a significant change in heart rate in groups $B$ and $C$, the values for time intervals are uncorrected for heart rate. PEP/LVET was calculated to the second decimal place. Stroke work index was obtained according to the following formula:

$$
\text { SWI }=\frac{(\text { SAm }-\mathrm{LAm}) \times \mathrm{SI} \times 13.6 \times \mathrm{I} \cdot 05}{100}
$$

Where SWI $=$ Stroke work index $\left(\mathrm{g} \mathrm{m} /\right.$ beat per $\left.\mathrm{m}^{2}\right)$ $\mathrm{SAm}=$ Systemic arterial mean pressure $(\mathrm{mmHg})$ 


$$
\begin{aligned}
\mathrm{LAm} & =\text { Left atrial mean pressure }(\mathrm{mmHg}) \\
\mathrm{SI} & =\text { Stroke index }\left(\mathrm{ml} / \text { beat per } \mathrm{m}^{2}\right) \\
13 \cdot 6 & =\text { Conversion factor from } \mathrm{mmHg} \\
& \mathrm{mmH}_{2} \mathrm{O} \\
\mathrm{I} \cdot 05 & =\text { Specific gravity of the blood }
\end{aligned}
$$

Pulmonary artery diastolic pressure (PAD), which was almost identical to the pulmonary wedge pressure in the control state in all patients, was used as an index of the mean left atrial pressure. The data were statistically analysed using Student's $t$ test.

\section{Results}

The results obtained in three groups of patients are presented in Table I. Cuff inflation in group A patients resulted in a significant increase in PEP associated with a decrease in LVET. Thus, the PEP/LVET ratio increased significantly. There was a significant fall in pulmonary artery systolic and diastolic pressure, stroke index, and stroke work index, while systemic arterial pressures did not change appreciably.

The responses of systolic time intervals to cuff inflation in patients in group B were strikingly different. PEP decreased significantly and LVET remained unchanged, resulting in a decrease in PEP/LVET ratio. Though pulmonary artery pressure fell as in group A, stroke index and stroke work index were not significantly changed.

Group $C$ patients did not manifest a change in either the systolic time intervals or the other haemodynamic parameters after cuff inflation.

As shown in Table 2, changes in PEP, LVET, PEP/LVET ratio, and PAD pressure resulting from cuff inflation were significantly different $(P<0.01)$ between groups $A$ and $B$. Difference between changes in stroke work index in these two groups was, however, of less significance $(P<0.05)$. Comparing

TABLE I Effects of cuff inflation on systolic time intervals and haemodynamics in 17 patients: mean values and standard error of mean

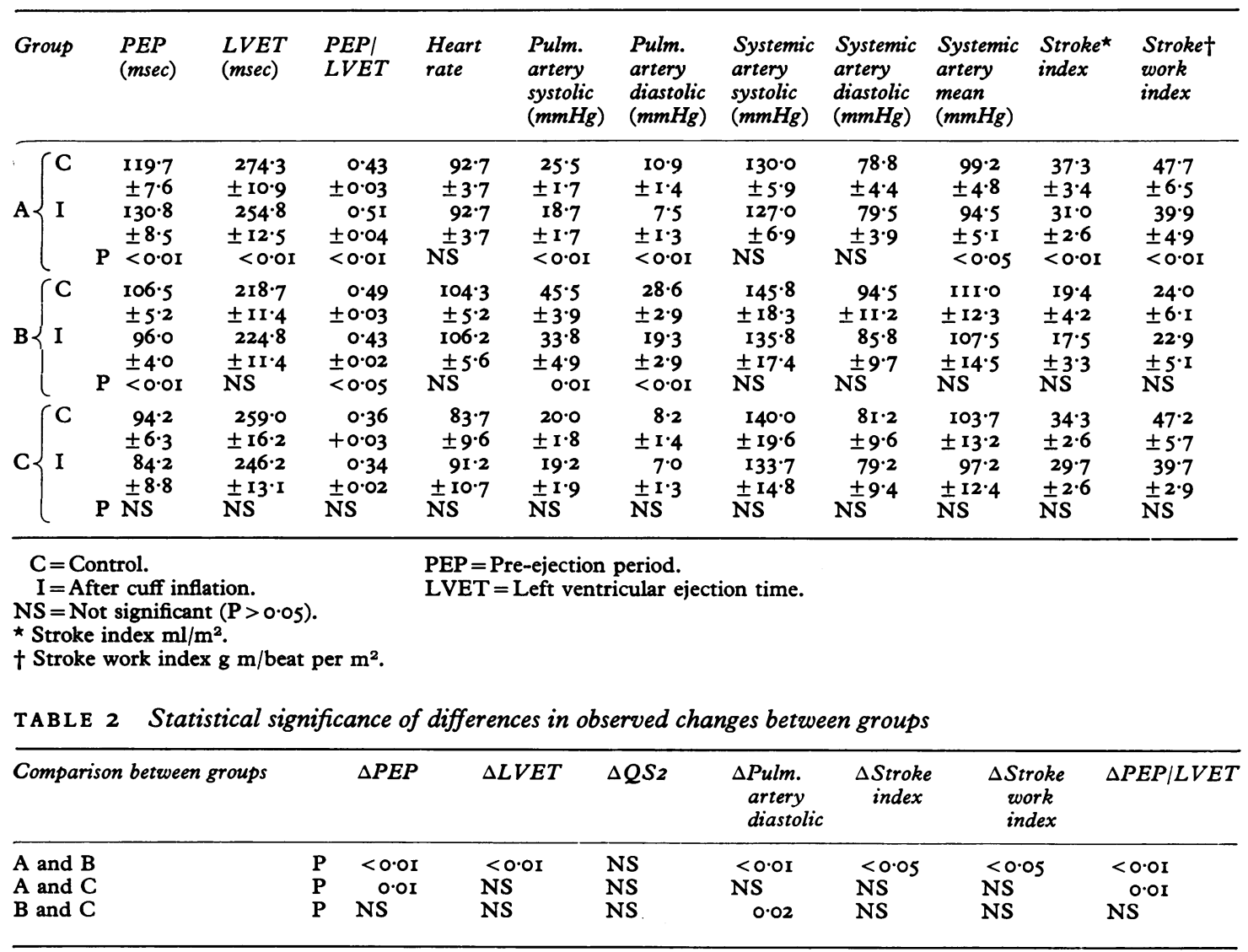

$\Delta=$ Change following cuff inflation. 
groups $\mathbf{A}$ and $\mathrm{C}$, a significant difference was observed only in PEP and PEP/LVET $(P<0.01)$.

When PAD was plotted against PEP (Fig. I), and also against PEP/LVET ratio (Fig. 2), there was a difference between patients in group $A$ from those in group B. Whereas a fall in PAD was associated with increase in PEP and PEP/LVET ratio in group A patients, PEP and PEP/LVET decreased in group $B$. The standard intervention produced no change in any of the parameters in group $C$. When stroke work index and stroke index were plotted against PAD (Fig. 3), a decrease in preload, as reflected in a fall in pulmonary artery diastolic pressure, resulted in a shift of the point to the left and downward in group $A$ and $C$ patients; while in group B the point moved straight to the left showing a striking reduction in pulmonary artery diastolic with no change in stroke index or stroke work index.

\section{Discussion}

Weissler, Harris, and Schoenfeld (1969) have correlated systolic time intervals with left ventricular function in man. They developed regression equations to allow for the effects of heart rate and studied the changes in these intervals after acute pharmacological interventions (Weissler et al., 196I). Patients with heart failure had significantly shortened LVET and prolonged PEP (Weissler, Harris, and Schoenfeld, 1968). Their observations of inverse correlations between the PEP/LVET ratio and left ventricular ejection fraction obtained angiographically suggest that the ratio may be used reliably without correction for heart rate (Weissler et al., 1969). The systolic time intervals have been correlated with direct measurement of left ventricular performance (Metzger et al., 1970). Widespread use of these intervals in valvular as well as in nonvalvular heart disease has been reported (Benchimol, Dimond, and Shen, 1960; Moskowitz and Wechsler, 1965; Harris, Schoenfeld, and Weissler, I967). It is generally recognized that despite a good correlation between systolic time intervals and ventricular dysfunction in groups of patients, there is considerable overlap in baseline values in individual patients (Hodges et al., 1972). Except for the extreme range of abnormal values, it is difficult to use systolic time intervals in a given patient to evaluate function or assess prognosis.

Our previous work (Shah et al., 1969) emphasized the role of alterations in preload on systolic time intervals, and we suggested that in the use of these intervals factors affecting preload, afterload, and contractility should be taken into consideration rather than comparisons of absolute values corrected

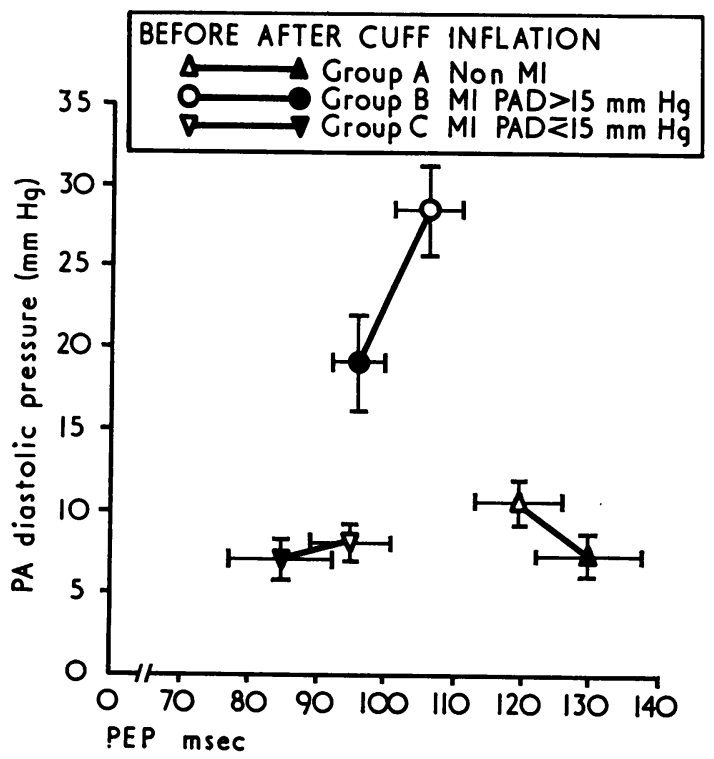

FIG. I Effects of venous cuff inflation on PEP correlated with pulmonary artery diastolic pressures in the three groups of patients. Mean values and $S E M$ are shown for each group. Directional changes in groups $A$ and $B$ were statistically different. Group $C$ patients showed considerable overlap and little directional changes.

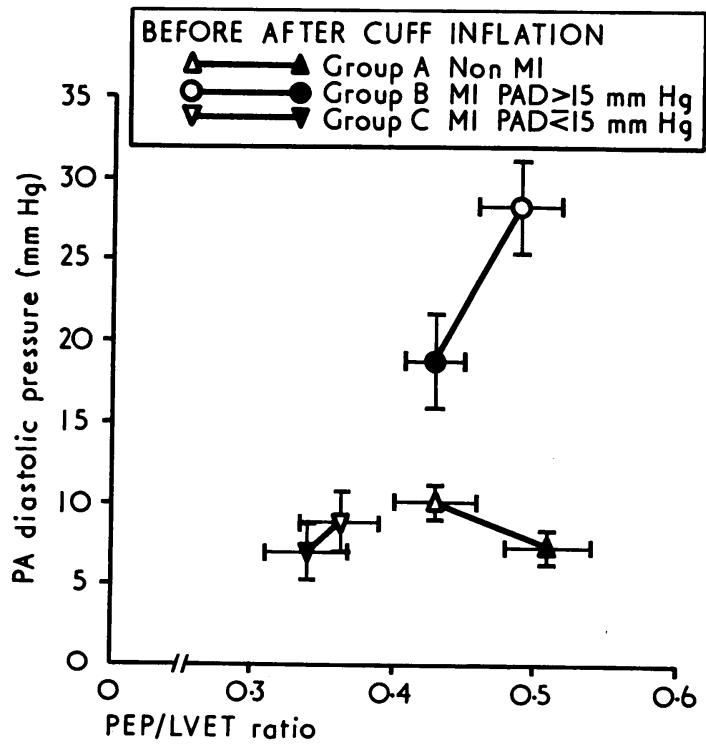

FIG. 2 PEP/LVET ratio correlated to pulmonary artery diastolic pressure before and after inflation of venous occlusive cuffs. A reduction in pulmonary artery diastolic pressure was associated with an increase in PEP/LVET ratio in group $A$, a decrease in group $B$, and little or no change in group $C$. Mean values with SEM are shown. 

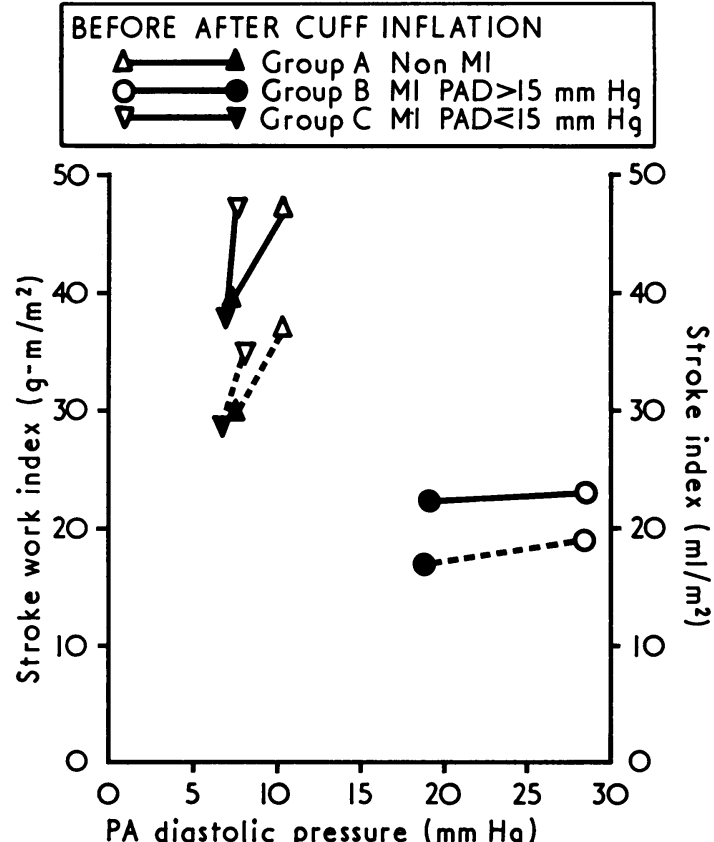

FIG. 3 Effects of venous cuffs on the relation between pulmonary artery diastolic pressure with stroke work index (continuous lines) and with stroke index (dashed lines) are illustrated for three groups of patients with their mean values. These may be viewed as Frank-Starling function curves and demonstrate appropriate drop in both pulmonary artery diastolic pressures and stroke work index as well as stroke index in group $A$ and $C$ patients. In contrast there was a striking reduction in pulmonary artery diastolic pressures associated with little change in stroke work index and stroke index in group $B$ patients. The changes in group $B$ patients may be considered a shift on the flat part of the ventricular function curve.

only for heart rate. The present study is an extension of this concept to patients with acute myocardial infarction. During venous occlusion by applying a tourniquet to the thighs, patients without acute myocardial infarction and having normal circulatory haemodynamics showed a prolongation of PEP, a decrease in LVET, and an increased PEP/LVET ratio. In striking contrast, patients with acute myocardial infarction having raised LV filling pressures showed a decrease in PEP and PEP/LVET ratio. Patients with acute myocardial infarction and normal filling pressures differed from either group and responded with no significant change either in PEP or LVET. In the present study, occlusive cuffs were applied on both thighs to obtain a decrease in venous return. This inter- vention was uniformly carried out in each patient and no attempt was made to quantitate changes $\mathbf{n}$ preload from alterations in pulmonary artery diastolic pressure.

The 'normal' response of prolongation of PEP with decreased venous return may be due to a reduction in the left ventricular end-diastolic volume as well as in the end-diastolic pressure resulting in a fall in the rate of development of left ventricular pressure (Gauer and Thron, 1965; Wang, Marshall and Shepherd, I960; Paley, Weissler, and Schoenfeld, 1964; Tuckman and Shillingford, 1966). A decrease in LVET is probably related to a fall in stroke volume, since the heart rate remained constant. An increased PEP/LVET ratio indicative of a fall in ejection fraction reflects a change in left ventricular performance as a result of reduced venous return. This is associated with an expected fall in cardiac output. Class I postsurgery patients with normal resting pressures manifested a 'normal' response to venous cuffs. Stafford et al. (1970) have observed a similar response after vertical tilt in normal subjects.

The 'abnormal' response in acute myocardial infarction patients with raised left ventricular filling pressures also may be explained on the basis of altered left ventricular performance. Under these conditions, the left ventricle responds differently to a reduction in preload. This is substantiated by the obervation that there was no significant change in the cardiac output despite a substantial fall in filling pressure. The left ventricle is thus functioning on the flat part of a depressed Frank-Starling function curve, so that reduction in end-diastolic pressure (and presumably end-diastolic volume) is not associated with a fall in stroke volume. Reduction in ventricular volume with a reduction in wall tension may result in a substantial drop in myocardial oxygen requirements and thus result in functional improvement of the ischaemic areas. A decrease in preload is likely to be much more beneficial for heart failure associated with coronary artery disease.

The response of acute myocardial infarction patients with normal ventricular filling pressures is difficult to interpret since no change in pulmonary artery diastolic pressure was observed. This may indicate a constricted venous bed under catecholamine effects (Lewis et al., 197I), and thus venous occlusive cuffs on the two thighs did not result in significant changes in venous return.

Comparisons of systolic time intervals in the control state showed considerable overlap between the three groups of patients. The group of patients referred to as 'normal' had aortic valve prostheses and despite normal resting circulatory haemo- 
dynamics, these do not represent normal healthy subjects. However, this study emphasizes a change in systolic time intervals after an acute intervention (i.e. application of venous occlusive cuffs); and the changes induced in this group are directionally different from acute myocardial infarction with raised LV filling pressures. Somewhat prolonged values for PEP in this 'normal' group represent prolongation of $\mathrm{QS}_{2}$ in patients with Starr-Edwards valve prostheses. Patients with acute myocardial infarction and normal left ventricular filling pressures had shorter PEP and lower PEP/LVET ratio. These changes are probably related to the increased catecholamine secretion observed after acute myocardial infarction. It is of some interest that acute myocardial infarction patients with raised left ventricular filling pressures had slightly longer PEP but considerably shorter LVET with increase of PEP/LVET ratio. The shorter LVET in this group is probably a reflection of a smaller stroke volume. The PEP/LVET ratio, however, was in the 'normal' range in many patients. Thus, in this study the control values of systolic time intervals appeared to be poor indicators of left ventricular performance in the presence of acute myocardial infarction.

It is proposed that in patients with acute myocardial infarction a decrease of PEP and PEP/ LVET ratio after acute reduction in preload indicates the presence of left ventricular dysfunction, i.e. an increase of pulmonary artery diastolic pressure greater than $15 \mathrm{mmHg}$. Furthermore, if pulmonary artery diastolic pressure is monitored and shows a significant drop along with decrease in PEP and PEP/LVET ratio, the depressed LV function is probably on the flat part of the Frank-Starling function curves. This response may be used to anticipate haemodynamic effects of acute diuresis. If these observations on venous occlusive cuffs on the systolic time intervals in acute myocardial infarction are confirmed by further studies, the responses may provide a simple potentially useful method to assess left ventricular function and to predict effects of acute diuresis in the early course of acute myocardial infarction.

We are deeply appreciative of Drs. Paul N. Yu, Bernard F. Schreiner, Jr., and Morrison Hodges for their help and co-operation. We thank Dr. Edward J. Dudweicz for assistance in statistical analysis of data.

\section{References}

Benchimol, A., Dimond, E. G., and Shen, Y. (1960). Ejection time in aortic stenosis and mitral stenosis. American Fournal of Cardiology, 5, 728.

Bennett, E. D., Smithen, C. S., and Sowton, G. E. (1970). Systolic time intervals in acute myocardial infarction (abstract). American fournal of Cardiology, 26, 625.
Diamant, B., and Killip, T. (1970). Indirect assessment of left ventricular performance in acute myocardial infarction. Circulation, 42, 579.

Garrard, C. L., Jr., Weissler, A. M., and Dodge, H. T. (1970). The relationship of alterations in systolic time intervals to ejection fraction in patients with cardiac disease. Circulation, 42, 455.

Gauer, O. H., and Thron, H. L. (1965). Postural changes in the circulation. In Handbook of Physiology Section 2, Circulation, Vol. 3, p. 2409. Ed. by W. F. Hamilton and P. Dow. American Physiological Society, Washington D.C.

Habte-Michael, A., Gould, L., and Gomprecht, R. F. (I97I). Systolic time intervals in myocardial infarction. Clinical Research, 19, 318.

Harris, W. S., Schoenfeld, C. D., and Weissler, A. M. (1967). Effects of adrenergic receptor activation and blockade on the systolic pre-ejection period, heart rate, and arterial pressure in man. Fournal of Clinical Investigation, 46, I704.

Hodges, M., Halpern, B. L., Friesinger, G. C., and Dagenais, G. R. (1972). Left ventricular pre-ejection period and ejection time in patients with acute myocardial infarction. Circulation, 45, 933.

Lewis, R. P., Boudoulas, H., McCabe, W. C., and Weissler, A. M. (I97I). Excessive adrenergic stimulation manifest by short systolic time intervals in acute myocardial infarction. Clinical Research, 19, 325.

Metzger, C. C., Chough, C. B., Kroetz, F. W., and Leonard, J. J. (1970). True isovolumic contraction time; its correlation with two external indexes of ventricular performance. American fournal of Cardiology, 25, 434.

Moskowitz, R. L., and Wechsler, B. M. (1965). Left ventricular ejection time in aortic and mitral valve disease. American fournal of Cardiology, 15, 809.

Paley, H. W., Weissler, A. M., and Schoenfeld, C. D. (1964). The effect of upright posture on left ventricular volume in man. Clinical Research, 12, 105.

Samson, R. (1970). Changes in systolic time intervals in acute myocardial infarction. British Heart fournal, 32, 839.

Seifen, E., Flacke, W., and Alper, M. H. (1964). Effects of calcium on isolated mammalian heart. American fournal of Physiology, 207, 716.

Shah, P. M., Tager, I. B., Schaefer, R. A., and Kramer, D. H. (1969). Determinants of pre-ejection period (PEP) of left ventricle in the assessment of myocardial performance in man. Clinical Research, 17, 263.

Shiner, P. T., Harris, W. S., and Weissler, A. M. (1969). Effects of acute changes in serum calcium levels on the systolic time intervals in man. American fournal of Cardiology, 24, 42.

Stafford, R. W., Harris, W. S., and Weissler, A. M. (1970). Left ventricular systolic time intervals as indices of postural circulatory stress in man. Circulation, 41, 485 .

Tuckman, J., and Shillingford, J. (1966). Effect of different degrees of tilt on cardiac output, heart rate, and blood pressure in normal man. British Heart fournal, 28, 32.

Wang, Y., Marshall, R. J., and Shepherd, J. T. (1960). The effect of changes in posture and of graded exercise on stroke volume in man. Fournal of Clinical Investigation, 39, IO5I.

Weissler, A. M., Harris, W. S., and Schoenfeld, C. D. (1968). Systolic time intervals in heart failure in man. Circulation, 37, 149. 
Weissler, A. M., Harris, W. S., and Schoenfeld, C. D. (I969). Bedside technics for the evaluation of ventricular function in man. American fournal of Cardiology, 23, 577.

Weissler, A. M., Kamen, A. R., Bornstein, R. S., Schoenfeld, C. D., and Cohen, S. (1965). The effect of deslanoside on the duration of the phases of ventricular systole in man. American fournal of Cardiology, 15, 153.
Weissler, A. M., Peeler, R. G., and Roehll, W. H. (I96I). Relationships between left ventricular ejection time, stroke volume, and heart rate in normal individuals and patients with cardiovascular disease. American Heart fournal, 62, 367.

Requests for reprints to Dr. Pravin M. Shah, Cardiology Unit, Strong Memorial Hospital, 260 Crittenden Blvd., Rochester, New York 14642, U.S.A. 